\title{
Collaboration between dentists and otorhinolaryngologists in treating temporomandibular joint syndromes
}

\author{
Christiana Madjova', Simeon Chokanov', Mario Milkov² \\ Department of Conservative Dentistry and Oral Pathology' \\ Department of Dental Materials Science and Propaedeutics of Prosthetic Dental Medicine 2 \\ Faculty of Dental Medicine, Medical University - Varna, Bulgaria
}

\section{Abstract}

Introduction:

The mandibular articulation is a complex system involving muscles, tendons, ligaments and innervation and it can cause pain or discomfort that should not be overlooked. Pathological changes in the joint can reduce the patient's quality of life by affecting his ability to eat and speak.

Objectives:

The aim of this study is to show that the collaboration between dentists and otorhinolaryngologists is the basis for good treatment of temporomandibular joint malfunctions.

Materials and methods:

A retrospective cross-sectional study was performed in patients with signs and symptoms of temporomandibular joint pain syndrome from 2018 to 2021 year. A total of 23 patients with clinical features consistent with TMS were enrolled. Patients were referred to dentists by otorhinolaryngologists.

\section{Results:}

The age range of patients was 23-67 years, mean of $45.30 \pm 18.9$ years. We found that 12 of them had bruxism, 6 abrasion due to stress and squeezing and 5 were with tooth damage. Almost $87 \%$ of them (20 of 23) had pain in the joint and masticatory muscles, $6-$ clicking sounds, 3 - pain when opening mouth and chewing. Mouth opening was normal in 20 patients and 3 had impaired movement of the jaws. All patients were treated conservatively - soft foods, antiinflammatory drugs, $13,1 \%$ - occlusal spints, $21.7 \%$ - selective filing of the contact interferences, $65,2 \%$ - height adjustment with nonremovable structures. Along with the height adjustment we applied botulinum toxin injections and for pain - NSAIDs.

Conclusion:

Good collaboration is crucial for the diagnosis, quality and competent treatment of temporomandibular disorders, especially in patients with overlapping signs and symptoms.

Key words: temporomandibular joint syndromes (TMS), temporomandibular joint disorders, temporomandibular joint dysfunction (TMD), dentists, otorhynolaryngologists, treating

\section{Introduction}

Mandibular articulation is a complex system involving muscles, tendons, ligaments and innervation and it can cause pain or discomfort that should not be overlooked. Pathological changes in the joint can reduce the patient's quality of life by affecting his ability to eat and speak (4).

Temporomandibular joint syndromes (TMS) are a group of conditions that affect the jaw muscles, the temporomandibular joints and the nerves and cause pain and dysfunction of the jaw joint and the muscles that control jaw movement. Symptoms may appear on one or both sides of the face, head or jaw or develop after an injury. This type of syndrome affects women twice as often (10).

Etiology of this conditions can be: trauma, parafunctional habits, occlusal microtrauma, tooth damage, bruxism, bruxomania, bad posture, harmful psychosocial changes, stress, sleep disorders, arthritis, systemic predisposition, chewing gum (3). In some cases, etiological factors can act together.

Symptoms that TMS dysfunction can cause are: joint or muscle problems, pain radiating to the face, jaw and neck, otalgia, tinnitus, deafness, pain in the temple, headache, including migraine, stiffness of the jaw muscles, limited movement or inability to open the jaw, painful clicking or popping in the jaw, change in the way the upper and lower teeth occlude, pressure and ear clogging, dizziness and 
blurred vision $(3,10)$. Temporomandibular joint disorders include: Costen's syndrome; displacement of the temporomandibular joint; clicking ,jaw; pain syndrome of temporomandibular joint dysfunction (TMD) (1).

AIM: The aim of this study is to show that the collaboration between dentists and otorhinolaryngologists is the basis for good treatment of temporomandibular joint malfunctions.

\section{Materials and methods}

A retrospective cross-sectional study was performed in patients with signs and symptoms of temporomandibular joint pain syndrome from 2018 to 2021 year.

A total of 23 patients with clinical features consistent with TMS were enrolled. Patients were referred to dentists by otorhinolaryngologists. When selecting patients for treatment, we paid attention to the presence of jaw pain during chewing, swelling of the joints, parafunctional habits (erasing tooth surfaces, sucking the thumb or lips, chewing gum), the presence of bruxism and bruxomania, trismus, headache, clicking or squeezing sound and difficulty in opening the mouth.

Our purpose was to show how important is the collaboration between otorhinolaryngologists and dentists in treatment of TMJ malfunctions. Due to this teamwork we managed to treat these patients through occlusal adjustment.

\section{Results and discussion}

We used a following algorithm to assess the characteristics and origin of TMS:

- first, the patient is asked about the complaints he has - what is their location, nature, beginning and duration;

- asking for any factors that relieve or intensify the pain;

- if any treatment has been applied so far, by what specialist and what exactly has been made;

- a detailed medical and dental history is taken, paying attention to general systematic and psychologic assessment.

Then proceed to the dental examination. We look for signs of tooth wear caused by bruxism or squeezing as a result of stress. Clicking, popping, accompanied by difficult opening and closing may be observed. The masticatory muscles should be palpated.
The dental examination includes also an analysis of panoramic radiography for fractures, arthritis or dislocation of the disc.

Additional imaging studies such as computed tomography (CT) or magnetic resonance imaging (MRI) are usually required in more severe cases and structural abnormalities $(14,6)$.

In our study, patients who were referred for dental treatment had problems with occlusion. The age range was 23-67 years with a mean of $45.30 \pm 18.9$ years. We examined and treated 23 patients. 12 of them had bruxism, 6 reported that the abrasion of the teeth was due to stress and strong squeezing and 5 were with tooth damage (fig.1). 20 of examined patients had complaints about the joint and masticatory muscles - from mild to severe pain, 6 complained of clicking sounds, 3 had pain when opening their mouth and while chewing. Mouth opening was normal in 20 patients, and 3 had impaired movement of the jaws. There was more than one complaint from patients, which is why the results collectively show a higher number. All patients were treated conservatively (fig.2).

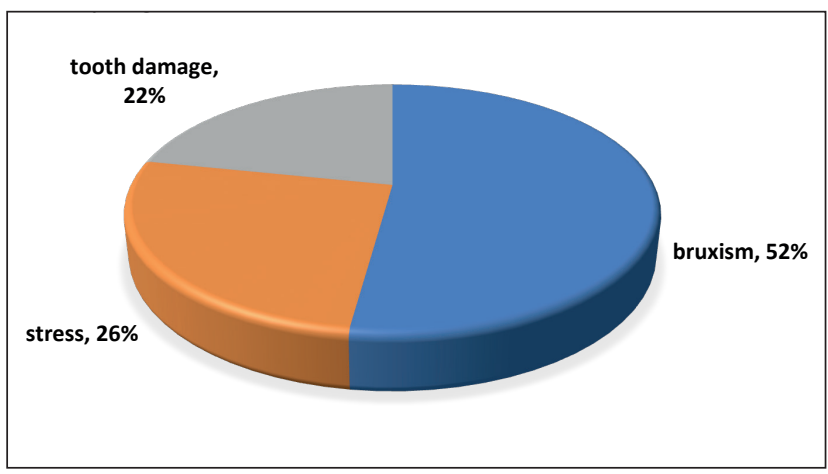

Fig.1. Patients, examined and treated with TMS from dentists

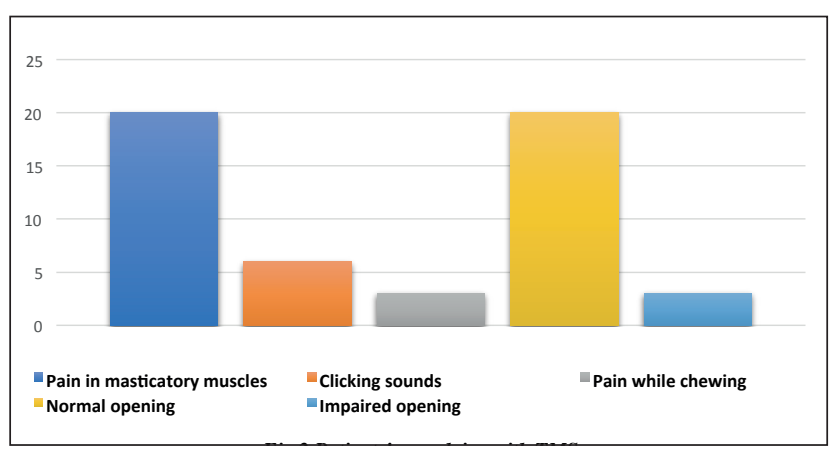

Fig.2. Patients' complains with TMS 
Our initial step in treatment was to advise patients to eat soft foods, prescribe anti-inflammatory drugs for acute pain and alternating hot and cold compresses.

In the study, $13,1 \%$ (3pts) were treated with occlusal spints. They were made individually by a dental technician to alter patients' occlusion. Other patients were treated by correcting the occlusion (irreversible occlusal therapy).

We applied selective filing of the contact interferences of the teeth in mild cases. In $21.7 \%$ (5) of patients which were in the initial phase of tooth wear responded well within a short period of time. In patients with more severe abrasion and lowering of the bite, a complete height adjustment was made with non-removable structures $(65,2 \%)$. Data is shown in table 1.

Along with the height adjustment we applied botulinum toxin injections for myorelaxation to 2 patients which were with chronic bruxism. For patients with impaired opening, severe pain in masticatory muscles and pain while chewing pharmacologic therapy were necessary. NSAIDs were prescribed for 14 days (Tabl.1).

Some studies show that occasionally, TMJ disorders may persist or recur (12). But so far we haven't seen in our treated patients any persistence or recurrence of symptoms.

It's known that the occlusion, respectively the temporomandibular joint, affects: the correct posture of the body (the muscles of the head and jaw are synchronized with the muscles from other parts of the body), body stability such as body balance, oscillation of the center of gravity and stability of vision and physical activity.

Temporomandibular joint dysfunction may also be accompanied by auditory symptoms such as otalgia, deafness, tinnitus, pressure / blockage and dizziness. Craniofacial pain is a symptom that is observed in temporomandibular disorders. It involves the joint, along with the masticatory muscles and the muscular nerves of the head and neck. Patients in the study were suffering from mild discomfort to unbearable pain.

Research data shows that TMD occurs in a higher percentage of people. It is most common in women between 20 and 40 years $(5,7)$. The percentage of people affected with at least one symptom varies from $10 \%$ to $39 \%$ according to the various studies $(5,7,8,9)$ but only about $5 \%$ are looking for treatment.

Very often, otorhinolaryngologists are the first doctors to get acquainted with the problem of the patients. Temporomandibular joint syndromes present a diagnostic challenge to the specialists, as orofacial pain, headache and otological symptoms are very common and mimic a number of otolaryngological conditions (13).

It is crucial to determine the etiology of the pain so that the right treatment can be applied. If the cause of TMS is a dental pathology, these patients should be reassigned. Thus a collaboration between a dentist and an otorhinolaryngologist is needed for better and more comprehensive treatment.

An undiagnosed temporomandibular joint disorder can lead to unnecessary investigation and treatment, leading to further patient suffering (13).

Before undertaking any treatment, TMS must be diagnosed very well, because sometimes there are diseases in temporomandibular joint area that mimic the condition. These can be dental caries, abscess, oral lesions (shingles, herpes simplex, oral ulcers, lichen planus), muscle spasms, bruxism, trauma, maxillary sinusitis, trigeminal neuralgia, salivary gland problems and others $(11,15)$.

\section{Conclusion}

Collaboration between healthcare professionals is

Tabl.1. Dental treatment in our patients according to the oral status

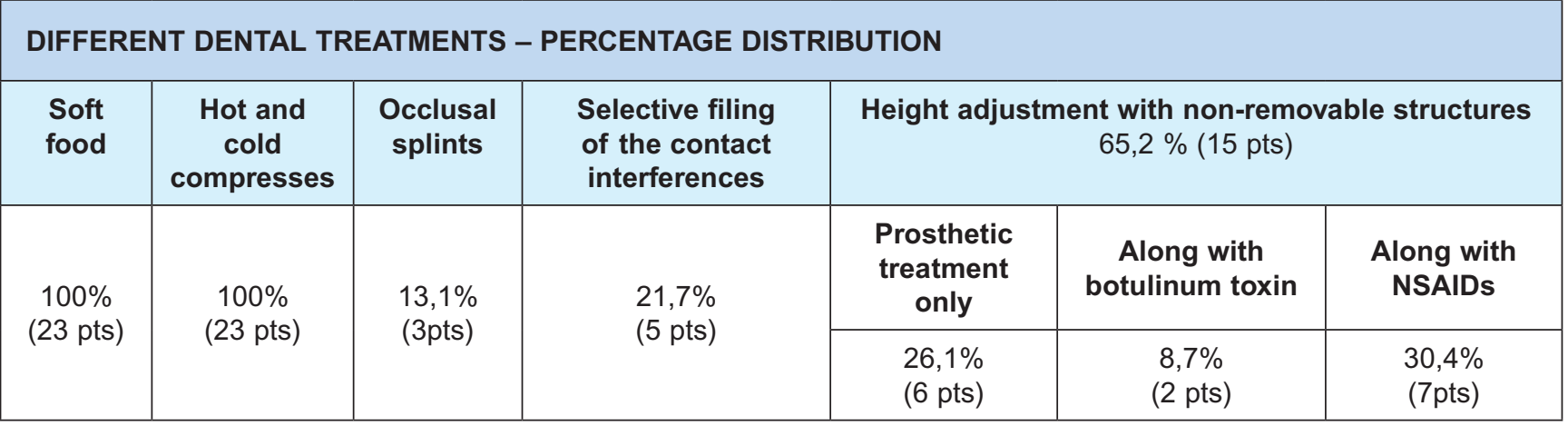


crucial for diagnosis, quality and competent treatment of temporomandibular disorders, especially in patients with overlapping signs and symptoms. Proper diagnosis and distinguishing the type of temporomandibular disorder from other otolaryngological conditions is the basis of effective treat- ment. Initial treatment can be initiated by an otolaryngologist by referring the patient to a dentist, oral and maxillofacial surgeon and a physiotherapist if needed.

Our patients responded very well to the complex treatments and returned their smiles.

\section{References:}

1. Ц Цинцовска Л. Нарушения на темпоромандибуларната става МКБ К07.6, 2014 https://medpedia.framar.bg/

2. American Dental Association (ADA). Temporomandibular Joint Syndrome, 2002

3. Cuhna J.14 Best Temporomandibular Joint Syndrome (TMJ) Treatments, MedicineNet

4. Gelb H., Calderone J., Gross S et al. The role of the dentist and the otolaryngologist in evaluating temporomandibular joint syndromes. The Journal of Prosthetic Dentistry; November 1967;18, (5): 497-503

5. Gonçalves DA, Camparis CM, Speciali JG et al. Temporomandibular disorders are differentially associated with headache diagnoses: a controlled study. Clin J Pain. 2011; 27 (7): 611-615.

6. Lewis EL, Dolwick MF, Abramowicz S et al. Contemporary imaging of the temporomandibular joint. Dent Clin North Am. 2008 Oct; 52 (4): 875-90, viii.

7. Lim PF, Smith S, Bhalang K et al. Development of temporomandibular disorders is associated with greater bodily pain experience. Clin J Pain. 2010; 26 (2): 116-120.

8. Liu F, Steinkeler A. Epidemiology, diagnosis, and treatment of temporomandibular disorders. Dent Clin North Am. 2013 Jul; 57 (3): $465-79$.

9. Maini K, Dua A. Temporomandibular Joint Syndrome. [Updated 2020 Nov 17]. In: StatPearls [Internet]. Treasure Island (FL): StatPearls Publishing; 2020 Jan. Available from: https:/www.ncbi.nlm.nih.gov/books/NBK551612/

10. NIH: National Institute of Dental and Craniofacial Research

11. Okeson JP, de Leeuw R. Differential diagnosis of temporomandibular disorders and other orofacial pain disorders. Dent Clin North Am. 2011; 55 (1): 105-120.

12. OM Eweka, OM Ogundana, GA Agbelusi. Temporomandibular pain dysfunction syndrome in patients attending Lagos university teaching hospital, Lagos, Nigeria. J West Afr Coll Surg. 2016 Jan-Mar; 6 (1): 70-87.

13. Stepan L., Shaw CK., Oue S. Temporomandibular disorder in otolaryngology: systematic review, The Journal of Laryngology \& Otology 2017; 131 (S1): S50-S56

14. Talmaceanu D, Lenghel LM, Bolog N et al. Imaging modalities for temporomandibular joint disorders: an update. Clujul Med. 2018 Jul; 91(3): 280-287.

15. Zakrzewska JM. Differential diagnosis of facial pain and guidelines for management. Br J Anaesth. $2013 ; 111$ (1): $95-104$.

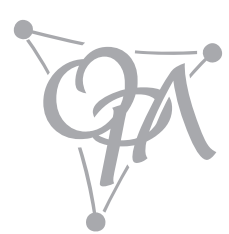

